\title{
Vorfeldbesetzung im Wirtschaftsdeutschen unter Anwendung des topologischen Satzmodells
}

\author{
Zdeněk VÁVRA
}

\begin{abstract}
Vorfeld-filling in business German using the topological sentence model

The author of this article analyses 50 articles from the field of business German and 50 texts written by students of business German. The article focuses on the filling of the Vorfeld (clause-initial position), using the topological sentence model developed by Drach. The following types of texts are researched: newspaper and journal articles, textbooks of business German, essays by students, and Bachelor theses. The author hypothesizes that the students fill the Vorfeld more often with the subject than is the case in the authentic German business texts, where adverbials and objects are also common in this position. Didactic methods are recommended which should help students to develop a command of natural sentence structures.
\end{abstract}

Keywords: business German, syntax, topological sentence model, Vorfeld-filling, didactic methods

DOI: doi.org/10.15452/StudiaGermanistica.2020.27.0006

\section{Einleitung}

Eine allgemein bekannte Besonderheit der deutschen Sprache besteht darin, dass die Teile des Prädikats als diskontinuierliche Konstituenten auftreten können. Eines der üblichen Instrumente zur syntaktischen Analyse, die diesem Tatbestand Rechnung trägt, stellt das topologische Satzmodell dar, das ausgehend von 1937 von Drach angestellten ersten Überlegungen in den folgenden Jahrzehnten systematisch ausgestaltet und weiterentwickelt wurde. Auf der Basis eines solchen Felder-Modells werden in dem vorliegenden Beitrag fünfzig Artikel aus dem Bereich des Wirtschaftsdeutschen auf die Regularitäten und Präferenzen bei der Besetzung der Vorfeldposition untersucht. Die dabei erzielten Ergebnisse werden in Bezug gesetzt zur Vorfeldbesetzung in fünfzig Texten von Studierenden vor und nach einer entsprechenden fachsprachorientierten Ausbildung.

Dass das an wirtschaftsbezogenen Texten untersucht wird, hat mit der praktischen Relevanz dieses Bereichs für die Studierenden zu tun. Die Fähigkeit zu angemessener schriftsprachlicher Kommunikation stellt gerade in den tschechisch-deutschen wirtschaftlichen Beziehungen eine wichtige Kompetenz dar. Nach wie vor besteht daher eine Nachfrage nach gut ausgebildeten Arbeitskräften, die zudem eine solide Kenntnis des Wirtschaftsdeutschen haben, da Deutschland „,mit einem Anteil von $29 \%$ " Tschechiens wichtigster Handelspartner bleibt (Wirtschaftsnachrichten 2020:6). Im Zuge der Corona-Krise hat zudem die Rolle der Korrespondenz per E-Mail im Wirtschaftsleben 
an Bedeutung gewonnen. Deshalb sollten die Studierenden in einschlägigen Fächern in die Lage versetzt werden, angemessen Wirtschaftsberichte, Stellungnahmen und Protokolle zu erstellen. Der Autor dieses Beitrags war sowohl in seiner beruflichen Funktion als Aufsichtsratsmitglied der kommunalen Betriebe mit diesen Anforderungen konfrontiert, und steht andererseits vor der Aufgabe, dem in seiner Unterrichtspraxis im Studiengang Fremdsprachen für Berufspraxis an der Westböhmischen Universität in Plzeň Rechnung zu tragen - unter anderem durch das Verfassen des elektronischen Lehrbuchs ,Hospodářská němčina (nejen) pro bakaláře‘, wo (z. B. im Kapitel 11) auch syntaktische Fragen behandelt werden (URL 3).

Die folgende Darstellung ist folgendermaßen aufgebaut, dass zunächst die Besonderheiten bei der Vorfeldbesetzung im Allgemeinen umrissen werden und dann an den fünfzig wirtschaftsorientierten Texten untersucht, welche Konstruktionstypen am häufigsten im Vorfeld stehen. Es zeigt sich im folgenden Vergleich mit fünfzig Texten der Studierenden, dass sie das Vorfeld häufiger mit dem Subjekt besetzen, als es in den authentischen deutschen Fachtexten der Fall ist, bei denen auch Adverbiale und Objekte mit einer gewissen Üblichkeit im Vorfeld stehen. Die fünfzig Texte des Wirtschaftsdeutschen, aus denen die Zahl der Satzglieder und die einzelnen Satzgliedtypen ermittelt werden, stammen teils aus der Fachpresse (25 Zeitungs- und Zeitschriftenartikel), teils aus Lehrbuchtexten (25 Artikel in Lehrbüchern des Wirtschaftsdeutschen). Bei den studentischen Vergleichskorpora handelt es sich um Aufsätze und Bachelorarbeiten von Studentinnen und Studenten des Wirtschaftsdeutschen des oben genannten Studiengangs. Im Anschluss an den Vergleich werden didaktische Methoden empfohlen, mittels derer bei den Studierenden des Wirtschaftsdeutschen die produktiven Fertigkeiten im Hinblick auf die mit der Vorfeldbesetzung verbundenen Fragen der Satzperspektive und die Fähigkeit zur Formulierung der üblichen Praxis angepasster Satzstrukturen gefördert werden sollen.

\section{Theoretische Ansätze und methodische Ausgangspunkte}

Im Fokus des topologischen Aufbaus des deutschen Satzes stehen Verbstellung, Stellungsfelder, Satzgliedfolge im Stellungsfeld, Partikel-Positionen und Herausstellungsstrukturen (Altmann/ Hahnemann 2010:27). In der funktionalen Grammatik wird der Satz als sprachliche Einheit in zweifacher charakterisiert, und zwar „1. als Einheit der Form und 2. als Einheit der Bedeutung“ (Schmidt 1977:132). Auch die Besetzung des Vorfelds lässt sich demnach sowohl unter formalem als auch unter semantischem Aspekt untersuchen. Unter formalem Aspekt können Themen wie die Struktur und die Eigenschaften des Vorfeldes, die Zahl der Elemente im Vorfeld (angemessen gefülltes vs. überfülltes Vorfeld) und Besetzung des Vorfeldes durch verschiedene Satzglieder untersucht werden.

Als Ausgangspunkt für die Analyse dient das Stellungsfeldermodell, das nach seinem Begründer in den 1930er Jahren auch Drach'sche Topologie genannt wird. Das Modell zielte darauf ab, die deutsche Grammatik von Denkweisen der lateinischen Strukturen zu lösen, das lebenswirkliche Sprechdenken zu beobachten und die Satzintonation in die Satzlehre einzugliedern. Diese Überlegungen wurden im weiteren Verlauf des 20. Jh. von mehreren Linguisten weiterentwickelt, beispielsweise von Johannes Erben, der sich mit verschiedenen Grundmodellen des Satzes befasste und sie schrittweise auf zwölf Baumodelle modifizierte, oder von Angelika Wöllstein, die u.a. Beschränkungen und Erweiterungen des topologischen Satzmodells wie Linksversetzung, Wackernagelposition und linke Satzperipherie aufgriff (vgl. Erben 1972:261 ff.; Wöllstein 2014:32-77).

Das Stellungsfeldermodell sagt zwar zunächst nichts über die hierarchische Strukturierung des Vorfeldes aus und ist nicht auf Sprachen ohne Klammerstruktur (wie das Englische oder Tschechische) anwendbar, aber da es gerade aufs Deutsche zugeschnitten ist, kann es im Hinblick auf seine Beschreibung umso mehr für relevant gehalten werden (Ramers 2007:67). Das Stellungsfeldermodell stellt anschaulich den topologischen Aufbau des deutschen Satzes dar, ist komplementär mit Klammerstrukturen und kombinierbar mit der Thema-Rhema-Gliederung, dem Bestandteil der Theorie der funktionalen Satzperspektive. Im Vergleich zum Englischen oder Französischen, d. h. zu (reinen) SVO-Sprachen, verdeutlicht das Stellungsfeldermodell, dass das Vorfeld im Deutschen 
nicht für das Subjekt reserviert ist; somit kann die daraus folgenden Variation auch für didaktische Zwecke (DaF/DaZ) von Bedeutung sein. Von der Relevanz des Stellungsfeldermodells zeugt die Tatsache, dass es als linguistisches Instrument seit mehr als 80 Jahren Anwendung findet, dass es in die Beschreibung der DUDEN-Grammatik Eingang gefunden hat und dass es um weitere Aspekte wie Aufteilung der Felder in Teilfelder oder Hinzunahme weiterer Felder vor dem Vorfeld ergänzt wird (vgl. Wöllstein 2014:53 ff.).

Unter semantischem Aspekt zählen zu den zu behandelnden Themen die kommunikative Aufgabe und der Mitteilungswert des Vorfeldes, die Stellung thematischer und rhematischer Konstituenten im Vorfeld und die Expressivität des Vorfeldes. Mit all diesen Punkten setzen sich theoretische Ansätze zur Topik-Kommentar-, Thema-Rhema-Gliederung oder als modifizierter Variante der Fokus-Hintergrund-Gliederung auseinander (vgl. Pittner/Berman 2015:142-144).

Für die Aufnahme von Texten in das analysierte Korpus wird eine Bestimmung des Wirtschaftsdeutschen vorausgesetzt. Für das Wirtschaftsdeutsche ist kennzeichnend, dass es eine enorme Heterogenität und eine relativ hohe Verflechtung mit dem Alltagsleben aufweist. Deswegen scheint in diesem Fall eine Definition nach dem Enumerationsprinzip günstiger zu sein als eine klassische Nominaldefinition. Dabei sind sowohl die horizontale als auch die vertikale Gliederung des Wirtschaftsdeutschen zu berücksichtigen. Während in der horizontalen Gliederung einzelne Bereiche wirtschaftlicher Aktivität mit ihren auch sprachlichen Eigenheiten nebeneinander stehen wie Wirtschaftswissenschaften, Wirtschaftspolitik, Industrie, Handel, Börse, Rechnungswesen u. v. a., folgt die vertikale Gliederung einzelnen Abstraktionsebenen innerhalb der wirtschaftlichen Kommunikation und überlappt teilweise mit der Hierarchie in einem Unternehmen. Die oberste Abstraktionsebene entspricht der Wissenschaftssprache, die mittlere Abstraktionsebene der Berufssprache des strategischen und operativen Managements und die niedrigste Abstraktionsebene der fachbezogenen Umgangssprache, die in der Geschäftskommunikation beispielsweise auf der Ausführungsebene im Büro oder im Betrieb verwendet wird.

Das Korpus, auf dem der vorliegende Beitrag beruht, umfasst Textmaterial aus den Jahren 2009-2019 von unterschiedlichen Ebenen des Wirtschaftsdeutschen. Neben deskriptiven Texten enthält das Korpus auch argumentative und instruktive Texte. Es handelt sich um 50 Artikel in Deutsch geschriebenen Fachzeitschriften wie das ,Handelsblatt' oder die ,Wirtschaftswoche“, Zeitungen (Rubrik, Wirtschaft') wie ,FAZ‘ oder ,Süddeutsche Zeitung' und Lehrbüchern des Wirtschaftsdeutschen (Niveau B2/C1). In das Textmaterial werden auch Unternehmenspräsentationen und Werbungen sowie Verträge eingegliedert. Neben diesen authentischen Texten wird die Vorfeldbesetzung in 50 Schularbeiten analysiert. Insgesamt werden folglich in 100 Texten unterschiedlicher Textsorten 1760 Satzglieder klassifiziert.

Im Hinblick auf das Ziel, die linguistische Analyse mit didaktischen Ansätzen und Implikationen für den konkreten Unterricht des Wirtschaftsdeutschen zu ergänzen, wird methodisch auf die Überlegungen von Comenius zurückgegriffen. Comenius verlangt Verknüpfung analytischer, synthetischer und synkritischer Methoden, wenn es um die vollständige Erkenntnis eines Sachverhaltes geht. Er vergleicht diese drei Methoden mit einem Fernglas, einem Vergrößerungsglas und einem Spiegel (Komenský 1946:58). Entsprechend werden hier zuerst auf der Basis der Diskussion in der Fachliteratur theoretische Aspekte des Vorfeldes im Allgemeinen besprochen, dann das authentische Textmaterial und die Schularbeiten (25 Aufsätze) zu Anfang des Bachelorstudiums des Wirtschaftsdeutschen (akademisches Jahr 2017/2018) in Bezug auf die Vorfeldbesetzung durch einzelne Satzglieder analysiert, letztlich die in den Schularbeiten auftretenden Abweichungen von der Wortfolge im authentischen Textmaterial identifiziert und Wege zur Aneignung einer realitätsnäheren Wortfolge am Satzanfang entworfen, d. h. didaktische Ansätze, die auf der empirisch ermittelten Verteilung der Satzglieder im Vorfeld in Fachzeitschriften und anderen authentischen Texten basieren.

Auf der Basis der so gewonnenen Erkenntnisse wurden didaktische und methodische Empfehlungen und Hilfsmittel entwickelt. Sie wurden im Deutschunterricht am Lehrstuhl für Germanistik und Slawistik der Westböhmischen Universität in Plzeň erprobt. Um ihre Wirkung zu beurteilen, wurden dann 25 ausgesuchte Kapitel aus den Bachelorarbeiten der Studierenden, deren Aufsätze 
aus dem ersten Studienjahr analysiert worden waren, untersucht. Diese Arbeiten wurden im dritten Studienjahr vor dem Abschluss des Bachelorstudiums (2019) verfasst und wieder hinsichtlich der Besetzung des Vorfeldes in einzelnen Sätzen untersucht. Letztlich werden die empirischen Daten von 2017 und 2019 miteinander verglichen und die Wirksamkeit der entworfenen Unterrichtshilfen ausgewertet.

\section{Eigenschaften des Vorfeldes im Allgemeinen}

Trotz formal unterschiedlichen Definitionen des Vorfeldes bei verschiedenen Linguisten ist der Kerngedanke im Prinzip identisch. Altmann/Hahnemann (2010:94) definieren das Vorfeld wie folgt: „Das Vorfeld erstreckt sich von der linken Satzgrenze bis zum Beginn des klammeröffnenden Elements. Die rechte Grenze ist bei Verb-Zweit das finite Verb, und zwar bei allen denkbaren Satztypen mit Verb-Zweit.“ Eine knappere Definition des Vorfeldes führen Habermann/Diewald/Thurmair (2015:94) an: „Das Vorfeld ist die Position vor dem finiten Verb eines Satzes, also vor der linken Satzklammer.“ Am kürzesten formuliert die Definition Sitta (1998:817): „Das Feld vor dem ersten Klammerteil nennt man Vorfeld.“

Vorfeldfähigkeit ist eines der Kriterien für den Status eines Elements als Satzglied (Altmann/ Hahnemann 2010:54). Wenn bei einer Satzart die Besetzung des Vorfelds vorgesehen ist, wie etwa im einfachen Aussagesatz, ist das, was im Vorfeld steht normalerweise ein Satzglied, ggf. auch in Form eines Nebensatzes oder einer Infinitivkonstruktion, bzw. ein Prädikatsteil. Auch die sogenannten Konjunktionaladverbien besitzen - im Unterschied zu Konjunktionen - Satzgliedstatus, wobei sie entweder im Vorfeld (Dennoch fahren wir fort) oder wie andere Satzglieder im Mittelfeld (Wir fahren dennoch fort; Beispiel nach Eisenberg 2001:228) stehen können. Von der grammatischen Notwendigkeit, die Vorfeldstelle zu besetzen, zeugt das sogenannte thematische es in Sätzen wie: Es ist erfrischend, am Abend spazieren zu gehen. Dieses Vorfeld-es stellt eine Ausnahme dar. Es hat keine Satzgliedfunktion, da es nicht erfragbar ist. Zudem fällt es bei einer Umstellung weg: Am Abend spazieren zu gehen ist erfrischend. Das unterscheidet dieses lediglich formale Subjekt, das auch als Platzhalter-es bezeichnet wird (Sitta 1998:629), von dem Scheinsubjekt es bei unpersönlich gebrauchten Verben (es regnet, es schneit, es klirrt u. Ä.) (Altmann/Hahnemann 2010:180). Mit dem Platzhalter-es wird nur die strukturell vorgesehene Stelle des Vorfelds in Verbzweitsätzen gefüllt, in denen die erste Stelle nicht besetzt ist (Sitta 1998:819).

Manche thematischen Teile am Satzanfang gehören hingegen nur scheinbar zum Vorfeld. „Extrapositionen oder Parenthesen, die nicht im Satzverband integriert sind, zählen natürlich nicht als Vorfeldbesetzungen“", betont Heringer (1996:249) und nennt das Beispiel: [Trotz chemotherapeutischer Behandlung], es wird langsam aber sicher Hautkrebs daraus. (Heringer 1996:249). In diesem Zusammenhang wird manchmal über das sogenannte Vor-Vorfeld gesprochen (vgl. Pittner/ Berman 2015:87). Diese Position ist nicht zuletzt für koordinierende Konjunktionen, ansonsten für linksversetzte und herausgestellte Konstituenten bestimmt. Auf die Problematik des Vor-Vorfeldes wird in diesem Beitrag nicht weiter eingegangen.

Im Vorfeld kann jedes Satzglied stehen, den Prädikatsteil einbegriffen: Gelogen hat er. Gewonnen haben wir nicht, aber auch nicht verloren! (Sitta 1998:820). Verschiedenen Untersuchungen zufolge ist das Vorfeld in mehr als 50 \% aller Fälle durch das Subjekt besetzt. Ohne weitere Präzisierung fügt Dürscheid (2007:97) hinzu, dass ,, auch Objekte und Adverbiale sehr häufig diese Position [einnehmen]“. Luscher (2001:25) in ihrer ,Übungsgrammatik für Ausländer" formuliert als Faustregel: „Im Deutschen stehen Angaben oder Ergänzungen oft am Satzanfang, zum Beispiel nach einer Frage.“ Auch ihre Formulierung, dass im Vorfeld „manchmal“ auch Objekte (Dativ- und Akkusativergänzungen) vorkämen (Luscher 2001:99) erscheint als ziemlich vage. Bei dieser Stellung der Objekte geht es in der Regel um einen Fall von Ausdrucksstellung, die mit der Besetzung des Mittelfeldes interagiert (Heringer 1996:250).

Die meisten Verbpartikeln kommen zwar im Vorfeld nicht vor, doch es gibt einige trennbare Präfixe, die heutzutage vorfeldfähig sind, wie Sittas (1998:820) Auffällt, dass keiner einen Anspruch 
anmeldet belegt. Auch weitere Fälle erscheinen: Fest steht, dass ..., Hinzu kommt, dass ..., Ab geht die Post. Was dagegen im Vorfeld überhaupt nicht vorkommt, sind Attribute: „Attribute stellen keine selbstständigen Stellungsglieder dar, sondern sind immer nur ein Teil eines Satzglieds, d.h. sie sind nicht allein vorfeldfähig, nicht allein herausstellbar" (Altmann/Hahnemann 2007:91). Nicht vorfeldfähig sind in der Standardsprache auch Modalpartikeln, das obligatorische Reflexivpronomen, die Satznegation nicht, der Dativus ethicus und es als Akkusativobjekt (Maroszová/Vachková 2009:55). Ihrer Funktion entsprechend sind Konstruktionen nicht vorfeldfähig, die sich obligatorisch auf einen vorangehenden Hauptsatz beziehen, wie Konsekutivsätze mit der Konjunktion sodass.

\section{Vorfeldbesetzung im Wirtschaftsdeutschen}

\subsection{Der formale Befund}

Eines der Ziele der Vorfeldanalyse in 50 ausgesuchten Fachartikeln war, festzustellen, ob in solchen Texten im Vorfeld auch mehrere Satzglieder stehen können, wie das beispielsweise in der literarischen Sprache gelegentlich der Fall ist.

Generell kann nach Auffassung mancher Linguisten im Vorfeld im Gegensatz zu anderen topologischen Feldern höchstens ein Satzglied stehen: „Das dem Verb Vorangehende (nach Drach das ,Vorfeld') ist grammatisch immer eine Einheit, d. h. ein Satzglied mit oder ohne Attribute“ (Jung 1968:97). Diese Einheit im Vorfeld kann (z. B. durch Nebenordnung) auch mehrgliedrig sein. In anderen Publikationen wird allgemeiner über eine Konstituente gesprochen: „Das Vorfeld darf dagegen nur durch eine Konstituente besetzt sein“ (Ramers 2007:66). Nach der Meinung von Habermann/Diewald/Thurmair (2015:95) könne das Vorfeld mit unterschiedlichen Elementen gefüllt sein, die aber jeweils ein Satzglied ausmachten: „Formal kann es sich um ein Wort, eine Phrase oder auch um Nebensätze handeln - aber alle diese Formen stellen genau ein Satzglied dar.“ Dürscheid (2007:101) formuliert diese Tatsache vorsichtiger: „Das Vorfeld wird normalerweise von einem Satzglied besetzt." Sie fügt jedoch hinzu, dass unter bestimmten Bedingungen das Vorfeld auch leer bleiben könne bzw. dass es auch mehrere Satzglieder aufnehmen könne.

Detaillierter beschäftigen sich mit der Zahl von Konstituenten im Vorfeld Pittner/Berman (2015:85-88). Die Titel der Kapitel 6.5 ,Besonderheiten bei der Vorfeldbesetzung: Ausnahmen von der Verbzweitregel?‘ und 6.6,Verbzweitstellung: Probleme bei der Identifizierung ' deuten an, dass in manchen Fällen nicht einfach zu unterscheiden ist, was eine Regel und was schon eine Ausnahme ist. Bei manchen Konstituenten handle es sich nur scheinbar um mehrere Satzglieder, beispielsweise könnte eine Apposition als Erweiterung eines Satzglieds betrachtet werden, oder Lokal- und Temporalangaben ließen sich miteinander zu einem komplexen Adverbial kombinieren. Da sie auch zusammen erfragbar sind, könnte man sie theoretisch auch für ein einziges Satzglied halten: Gestern im Kino nach dem Film hat sie ein Mann angesprochen. [...] Wann hat sie ein Mann angesprochen? Gestern im Kino nach dem Film (Pittner/Berman 2015:85). Uneinigkeit herrscht darüber, ob ein Satzglied mit einer Fokuspartikel oder einem Adverb zusammen eine Konstituente bilden. Pittner/ Bermann (2015:86) führen folgende Beispiele an: Nur (= Fokuspartikel) der Hans kam gestern nicht rechtzeitig. Hans leider (= Adverb) kannst du dort nicht antreffen. Die Stellung der Fokuspartikeln ist dabei relativ frei; sie können vor oder nach dem Bezugsausdruck (auch in der Distanzstellung) vorkommen: Nur ein Semester wird sie studieren. Ein Semester nur wird sie studieren. Ein Semester wird sie nur studieren (URL 1).

Um zwei unterschiedliche Satzglieder handelt es sich offensichtlich, wenn ein Objekt und ein Adverbial oder zwei (nicht zusammen erfragbare) Adverbiale im Vorfeld stehen. Diese Situation betrachten Pittner/Berman jedoch als „sehr selten“ (2015:86). Eine weniger strikte Auffassung vertritt Eisenberg (2001:389), vor allem in Bezug auf die markierte Wortfolge, die in literarischen Texten erwartet werden kann. Im Vergleich zu der unmarkierten Reihenfolge könne das Vorfeld bei der markierten Reihenfolge auch mit zwei oder sogar drei Satzgliedern besetzt sein: Ihm den Stern hat Irene gezeigt. Den Stern gezeigt hat Irene ihm. Ihm den Stern gezeigt hat Irene (Eisenberg 
2001:389). Da es sich bei den zitierten Beispielen aber um keine authentischen Beispiele handelt, ist ihre Aussagekraft beschränkt. In der Online-Sprachberatung der Universität Gießen wurde die Frage nach der Korrektheit des Satzes mit einem Objekt in Verbindung mit einem Adverbial im Vorfeld diskutiert. Das authentische Beispiel lautet: Einige News-Artikel schließlich haben wir gelöscht. Mit der Umstellprobe ist zu beweisen, dass das Adverbial schließlich das Vorfeld auch alleine besetzen kann, es somit vorfeldfähig ist: Schließlich haben wir einige News-Artikel gelöscht. Einige NewsArtikel haben wir schließlich gelöscht. Die Sprachberatung gibt jedoch keine eindeutige Antwort, ob sich im Vorfeld zwei Satzglieder befinden, und bietet mehrere Erklärungsmöglichkeiten, die von der Perspektive abhängig sind: 1. die laut Dudengrammatik immer noch regelkonforme Ausnahme (Objekt + Adverbial im Vorfeld); 2. ungrammatischer Satz (zwei Satzglieder im Vorfeld); 3. „Es handelt sich bei schließlich um einen Teil des Satzgliedes einige News-Artikel“ (URL 2). Im untersuchten Korpus trifft der Autor dieses Beitrags auf die Wortfolge mit mehreren Satzgliedern im Vorfeld nicht. In allen untersuchten Sätzen erscheint nur die unmarkierte Reihenfolge. Im Wirtschaftsdeutschen (wohl auch in anderen fachspezifischen Texten) sind so stark markierte Stellungen offenbar unüblich.

Auf der anderen Seite kann das Vorfeld unter bestimmten Bedingungen unbesetzt bleiben, „das Vorfeldelement [wird] getilgt" (Pittner/Berman 2015:86). Das erfolgt regelmäßig bei der Fortführung von beigeordneten Sätzen durch koordinierende Konjunktionen wie und, oder, nicht nur ... sondern auch. In bestimmten Kontexten - vor allem in Alltagsgesprächen, aber auch in literarischen Texten - kann ein unbetontes Vorfeldelement entfallen; dies betrifft vor allem entsprechende Pronomina im Nominativ oder Akkusativ (beispielsweise in Sätzen wie Kann sein. Kommt darauf an. Hab ich gehört). In den untersuchten schriftlichen Texten des Wirtschaftsdeutschen kommt nur die erste Möglichkeit des leeren Vorfelds, die Tilgung bei koordinierenden Konjunktionen, vor.

\subsection{Vorfeldbesetzung in den untersuchten Wirtschaftstexten}

Das nächste Ziel der Analyse bestand in der Ermittlung des prozentuellen Anteils der einzelnen Satzgliedtypen in den untersuchten Texten. Die Verberstsätze (Aufforderungssätze, Entscheidungsfragen usw.), bei denen kein Vorfeld vorhanden ist, wurden aus der Analyse ausgeschlossen. Aus demselben Grund wurden uneingeleitete Konditionalsätze nicht in Erwägung gezogen. Sie sollen in einer weiteren Studie behandelt werden, da sie in Wirtschaftstexten sprachökonomische Ziele erfüllen, wie in dem folgenden Beispielsatz: Rechne man diesen Effekt heraus, bleibe ein Wachstum von 1,3 Prozent (Bauchmüller 2019:1). Um Satzglieder ermitteln zu können, hat der Autor die klassischen operationalen Verfahren wie Frage-, Umstell-, Ersatz- und Weglassprobe durchgeführt. Daraus ergibt sich, dass in 50 analysierten Fachartikeln meistens das Subjekt (57,2 \%) im Vorfeld steht, gefolgt von Adverbialen (31,8 \%) und Objekten (7,2 \%). Auf Prädikatsteile, Prädikative und Vorfeld-es entfallen insgesamt lediglich 3,8 \% (1,4\%, 1,3\%, 1,1\%).

Die Artikel in Fachzeitschriften und Zeitungen (Rubrik: ,Wirtschaft') weisen einen höheren Anteil von Adverbialen (32,6\%) und Objekten im Vorfeld (8,0\%) auf als die Lehrbuchartikel (Adverbiale 30,5\%, Objekte 5,7 \%). Der Anteil von Subjekten im Vorfeld bei Fachpresseartikeln ist hingegen niedriger (55,4\%) als bei Lehrbüchern des Wirtschaftsdeutschen (60,4\%). Dies könnte darauf zurückzuführen sein, dass sich die Lehrbuchautoren zum Ziel setzen, die Texte möglichst leserfreundlich zu gestalten, weshalb sie häufiger die SVO-Wortfolge wählen und weniger übliche Satzglieder im Vorfeld eher vermeiden. Obwohl die Verteilung von Satzgliedern in diesen zwei Gruppen von Fachartikeln ähnlich ist, lässt sich bei den Autoren der Fachpresseartikel eine verstärkte Tendenz beobachten, das Vorfeld häufiger mit einem anderen Satzglied zu besetzen als mit dem Subjekt. Im Durchschnitt beginnen mehr als $40 \%$ der Fachpresseartikel mit einem Adverbial oder einem Objekt.

\section{Vorfeldbesetzung im Wirtschaftsdeutschen unter semantischem Aspekt}

In diesem Unterkapitel wird auf die kommunikative Aufgabe und auf den Mitteilungswert des Vorfeldes im Wirtschaftsdeutschen eingegangen. Von einer Anzahl der in der linguistischen Literatur zu 
findenden Erklärungen, welche Rolle das Vorfeld im kommunikativen Sinne spielen kann, werden an dieser Stelle vier Gruppen betrachtet.

1. Dem Vorfeld fällt bei einer neutralen Wortfolge die Aufgabe zu, ,die Verbindung zu den Gegebenheiten des voraufgehenden Textes oder der vorgegebenen Situation herzustellen“ (Weinrich 1993:40). Im Vorfeld steht bei der objektiven Abfolge der Anschluss an Gegebenes, d. h. das Thema, das zugleich einen Ausgangspunkt für das neu Mitzuteilende darstellt. Im Rahmen des von Daneš entwickelten Konzepts der thematischen Progression (Daneš 1974:106-128) werden dabei verschieden Progressiontypen unterschieden, so die einfache lineare thematische Progression $(\mathrm{T} 1 \rightarrow \mathrm{R} 1=\mathrm{T} 2 \rightarrow \mathrm{R} 2)$, die thematische Progression mit durchlaufendem Thema $(\mathrm{T} 1 \rightarrow \mathrm{R} 1$; $\mathrm{T} 1 \rightarrow \mathrm{R} 2)$ und die thematische Progression mit gespaltenem Thema ( $\rightarrow$ R1, R2, R3) (Guber 2004:31-34). Diese kommunikative Aufgabe des Vorfeldes tritt im Wirtschaftsdeutschen häufig auf: Für 2019 und 2020 sollen es zusammen 7,6 Millionen Euro sein. Das Geld stammt aus einer ohnehin zugesagten Fördersumme von 9,5 Millionen Euro für die nächsten fünf Jahre (Regionalflughäfen ... 2019:20).

2. Eine ähnliche Funktion erfüllt das Vorfeld, wenn darin etwas genannt wird, das zwar nicht an etwas Gesagtes anschließt, das aber als bekannt vorauszusetzen ist (,das pragmatische Vorwissen“) (Weinrich 1993:63). Auch in diesem Fall handelt es sich um keine Hervorhebung, sondern eher um eine Einführung in die Situation. Das Satzglied im Vorfeld wird in diesem Fall nicht eigens betont (ebenfalls wie ad 1.) (Sitta 1998:819). Das Rhema steht im Mittelfeld, ,und zwar häufig gegen das Ende des Satzes“" (Sitta 1998:820). Seine Anwendung findet dieses Verfahren im Wirtschaftsdeutschen insbesondere am Anfang der Zeitungsartikel als Aufhänger. So wird z. B. beinahe jeder Artikel in der Rubrik ,Unternehmen' in der ,Frankfurter Allgemeinen' mittels dieses Verfahrens eingeleitet: Die jüngsten Insolvenzen von Fluggesellschaften sorgen für weniger Verkehr an kleinen Flughäfen (Regionalflughäfen ... 2019:20 ff.). Durch ein angemessen thematisch gefülltes Vorfeld signalisiert der Journalist, dass er den Leser für eine Person hält, die über einschlägige Vorkenntnisse verfügt und wohl mehr von der Fachthematik erfahren möchte. Weniger leserfreundlich ist jedoch, wenn das Vorfeld (z. B. durch ein erweitertes Attribut) zu überfüllt ist: Der im vergangenen Herbst in Aussicht gestellte Verkauf des Geschäftsbereichs Flugzeugfinanzierungen durch den deutschen Verkehrsfinanzierer DVB Bank kommt gut voran (DVB ... 2019:20).

3. Das Vorfeld erfüllt seine kommunikative Aufgabe als eine Ausdrucksstelle. Fokussierte Elemente landen im Vorfeld, wenn der Autor sie hervorheben will oder wenn er dadurch vom Leser eine besondere Deutungsarbeit erwartet (Heringer 1996:254). Beneš (1977:122) spricht in diesem Kontext über das ,gefühls- oder willenswertige[s] Sinnwort". Für einen erregten Sprechablauf ist die subjektive Abfolge Rhema - Thema typisch: Schön ist das Wetter! [...] Schämen sollten Sie sich! (Beneš 1977:123-125). Bei einer Voranstellung eines infiniten Prädikatsteils im Vorfeld erhält der Satz einen emphatischen Ausdruckswert, was in der gesprochenen Sprache mit einem Intensitätsakzent einhergeht: Gelesen habe ich viel, geschrieben wenig (Weinrich 1993:75). Helbig/Buscha (2013:488) bemerken, dass die emphatische Wortstellung oft die freien Modalbestimmungen betreffe: Ich habe das Buch mit großem Interesse gelesen. $\rightarrow$ Mit großem Interesse habe ich das Buch gelesen. Ebenfalls kann eine Information durch das Objekt im Vorfeld hervorgehoben werden, da diese Stellung als eher ungewöhnlich wahrgenommen wird: Den Pokal hat wie immer Eva gewonnen (Habermann/Diewald/ Thurmair 2015:98). Diese Art der Stellung des Objektes im Vorfeld kam in 8,0 \% von allen analysierten Sätzen in Zeitungen (Rubrik, Wirtschaft') und Fachzeitschriften vor; in den analysierten Bachelorarbeiten fand sich in 6,8\% der Vorfeldbesetzungen diese Funktion, in Lehrbüchern des Wirtschaftsdeutschen in 5,7 \%, in Aufsätzen hingegen 
nur in 2,9\%) diese Funktion. Genannt sei ein Beispiel aus der Zeitschrift, Wirtschaftswoche': Einen echten Effekt haben die beruflichen Ausgaben allerdings nur, wenn 1000 Euro Gesamtsumme im Jahr überschritten werden - für alle beruflichen Kosten (Hoyer 2019:84). Wenn (betonte) Pronomina in der Objektrolle im Vorfeld die auftreten, dann haben sie rhematische Funktion. Dazu kommt es vorwiegend in umgangssprachlicher Kommunikation: Dem habe ich es aber deutlich gesagt! Die habe ich noch nie so zornig gesehen! (Weinrich 1993:388). Außerdem kann auf diese Weise ein Gegensatz angedeutet werden: Ihm habe ich das nicht gesagt, wohl seinem Bruder (Maroszová/ Vachková 2009:55). Die Verteilung von neuer und alter Information macht sich auch im Falle der Besetzung des Vorfelds durch einen Nebensatz bemerkbar: „Die Vorfeldbesetzung von Nebensätzen ist zur Stellung im Nachfeld nicht immer bedeutungsgleich“ (Habermann/Diewald/Thurmair 2015:119). Wenn der Nebensatz im Vorfeld steht, rückt das Subjekt im Hauptsatz hinter das Prädikat ins Mittelfeld, wobei es dann (bei kurzen Hauptsätzen) praktisch fast am Ende des ganzen Satzgefüges stehen und dadurch ein Rhema signalisieren kann. Die unterschiedliche satzfunktionale Akzentuierung soll hier an den folgenden zwei Sätzen veranschaulicht werden: Sie schrie auf, als sie die Spinne bemerkte. $\rightarrow$ Als sie die Spinne bemerkte, schrie sie auf. Im Wirtschaftsdeutschen ist jedoch die Platzierung einer hypotaktischen Konstruktion als eines Vordersatzes weniger üblich: In einer Ausgabe der Wirtschafts- und Finanzzeitung ,Handelsblatt‘ wurden im Zuge der Analyse von Artikeln in der Rubrik ,Meinung \& Analyse" insgesamt nur drei Vordersätze identifiziert. Es handelt sich um Sätze der folgenden Art: Bevor Airbnb mit seinen Betten in Privatwohnungen den Markt aufrollte, konnten Städte über die Zahl der Hotelbetten die Besucherströme halbwegs steuern (Louven 2019:26).

4. Das Vorfeld enthält das weniger Wichtige oder ist inhaltlich ganz entleert. Dadurch bleiben sowohl das die Stellung der verbalen Glieder bestimmende satzstrukturelle Prinzip als auch das die Stellung der nichtverbalen Glieder beeinflussende Prinzip der Satzperspektive eingehalten. Diese Prinzipien könnten sonst in Konflikt geraten, wenn der Autor eines Textes die Spannung erhöhen wolle (Beneš 1977:124). Um keines der beiden Prinzipien zu verletzen, kann das Vorfeld-es in Platzhalterfunktion eingesetzt werden, damit die Pointe an den Satzschluss oder in seine Nähe rückt: Es hätten sich ausreichende Anhaltspunkte für strafbares Verhalten ergeben, teilte die Staatsanwaltschaft in Den Haag mit (Shell-Manager ... 2019:20). Die zweite Möglichkeit besteht darin, dass der verbale Schlussteil der Satzklammer (ähnlich wie ad 3.) die Spitzenstellung einnimmt. Im Gegensatz zur Ausdrucksstellung erfährt der Leser am Satzanfang kaum eine neue Information, der überraschende Schlusseffekt kommt möglichst spät, wie im eigenen Beispiel des Autors der Fall ist: Hinzugekommen ist, dass er ein absoluter Kontrollfreak ist und seine Mitarbeiter psychisch terrorisiert.

\section{Vorfeldbesetzung in den studentischen Texten und einschlägige didaktische Ansätze}

2017 wurden 25 auf Deutsch geschriebene Aufsätze der Studentinnen und Studenten des Wirtschaftsdeutschen am Lehrstuhl für Germanistik und Slawistik der Westböhmischen Universität in Plzeň in Bezug auf die Vorfeldbesetzung untersucht. Es handelte sich um Studierende in der Anfangsphase des Bachelorstudiums, die u. a. die Seminare Syntax der deutschen Sprache, Wirtschaftsdeutsch oder deutsche Handelskorrespondenz besucht haben.

Bei der Untersuchung stellte sich als erstes heraus, dass in diesen Aufsätzen bei mehr als zwei Dritteln der Sätze $(68,6$ \%) das Subjekt im Vorfeld stand. In manchen Aufsätzen kommt sogar dasselbe Subjekt immer wieder vor. Im Gegensatz zu einem instruktiven Text wirken in einem informativen Text die Sätze mit einem wiederholten Subjekt im Vorfeld störend: 
Es gibt viele Möglichkeiten, wie man die Erhöhung der Rohstoffpreise als Produzent lösen kann. Der Produzent kann zum Beispiel alternative Rohstoffe nutzen. Der Produzent kann neue Produkte ohne die teuren Rohstoffe herstellen. Der Produzent kann mit einem anderen Unternehmen (als JointVenture) kooperieren. Der Produzent kann sich auf die Bio-Landwirtschaft konzentrieren, weil die Kunden auf Qualität achten. (Čechová 2017:1)

Wenn die Studierenden so in der Anfangsphase des Bachelorstudiums bevorzugt das Subjekt im Vorfeld verwenden, so liegen sie damit um 13,2 \% über dem Durchschnitt der als Vergleichsbasis gewählten Artikel aus der Fachpresse. In 23,4 \% der von Studierenden produzierten Sätze stand im Vorfeld ein Adverbial und nur in 2,9 \% ein Objekt. Über die Gründe, warum manche Pilsner Studierende in dieser Phase ihres Studiums das Vorfeld so weitgehend mit dem Subjekt besetzen, lässt sich nur spekulieren: Wohl hat das Englische als die häufigste an allgemeinbildenden Schulen in Tschechien gelehrte Fremdsprache Einfluss auf die niedrigere Sprachkompetenz in Deutsch, die wiederum die Wahl der Mittel in diesem Bereich begrenzt. Ziel der fachsprachlichen Ausbildung sollte es daher sein, durch die Vermittlung von verschiedenen Varianten der syntaktischen Gestaltung eine höhere Abwechslung in der Formulierung und eine adäquatere Textgestaltung zu ermöglichen. Die folgende Umformulierung des obigen Textes durch den Autor dieses Beitrags zeigt, dass in es diesem Fall im Hinblick auf die oben beschriebenen Typen, ggf. auch auf die damit verbundenen thematischen Progressionstypen möglich und sinnvoll ist, das Subjekt im Vorfeld nicht zu wiederholen:

Es gibt viele Wege, wie ein Unternehmen den Anstieg der Rohstoffpreise bekämpfen kann. Am einfachsten wäre, die Produkte mit Haselnüssen aus dem Sortiment zu nehmen. In diesem Fall wird sicherlich die Konkurrenz die Marktlücke ausnutzen. Da die Kunden auf Qualität achten, könnte auch die bäuerliche Landwirtschaft eine Option sein. Hilfreich wäre einen neuen Lieferanten zu finden, um sich aus der Abhängigkeit vom Quasimonopolisten zu befreien.

Am Beginn dieser Untersuchung wurde angenommen, dass die Studierenden im Laufe des Hochschulstudiums infolge der Auseinandersetzung mit authentischen Texten, das Vorfeld im Laufe der Zeit zunehmend nicht mehr primär mit dem Subjekt, sondern auch verstärkt mit anderen Satzgliedtypen besetzen würden. Diese Hypothese ließ sich auf der Basis der im Jahr 2019 am Ende des Bachelorstudiums von denselben 25 Studentinnen und Studenten geschriebenen Texte verifizieren. Wie zu vermuten war, war der Anteil der im Vorfeld vorkommenden Subjekte in den Bachelorarbeiten niedriger als in den Aufsätzen vom Studienbeginn. Im Vorfeld stand bei den Testpersonen des dritten Studienjahres zwar ebenfalls noch am häufigsten das Subjekt, aber sein Vorkommen ist um $17,1 \%$ auf 51,5\% gesunken. Entsprechend nahm die Besetzung des Vorfelds mit Adverbialen und Objekten zu. Die Verwendung von Adverbialen im Vorfeld ist um 13,7 \% auf 37,1 \% gestiegen, die Anzahl der Objekte hat sich um 3,9 \% auf 6,8 \% erhöht. Welche Folgen das für die Textgestaltung hat, zeigt der folgende Auszug aus einer 2019 verteidigten Bachelorarbeit:

Das Hauptziel des theoretischen Teils war es, das internationale Marketing, die Marketingkommunikation sowie die Messen und Ausstellungen allgemein zu beschreiben und ihre Prinzipien zu erklären. Dem Leser wurden auch die Grundbegriffe aus diesem Bereich und Informationen aus dem wirtschaftlichen Umfeld nähergebracht. In dem praktischen Teil hat sich die Autorin auf mehrere Aspekte konzentriert. Zuerst hat sie die historischen Tatsachen untersucht. Dann hat sie fünf konkrete Messen aus Bayern und eine aus Tschechien genannt und einzeln vorgestellt. Für ihre Interviews hat sie sich bemüht die westböhmischen Unternehmen zu finden. (Čapková 2019:45)

Auf den ersten Blick ist zu erkennen, dass nicht nur die Möglichkeiten der Variation bei der Vorfeldbesetzung in erhöhtem Ausmaß genutzt werden, sondern, dass durch die Schulung an authentischen Texten eine erheblich höhere Texttypadäquatheit erreicht wurde.

Damit bestätigen sich die Ergebnisse der Dissertation des Verfassers (Karlsuniversität 2013), dass der Unterricht mit authentischen Materialien (UE B) effektiver ist als der mit Texten, die für den Unterricht eigens erstellt oder zu diesem Zweck modifiziert wurden (UE A). Das schlägt sich auch im Index der pädagogischen Polarität nieder (vgl. Pelikán 2014: 216-17). Im Gegensatz 
zu vereinfachten und didaktisierten Texten führen authentische Materialien demnach trotz eines höheren Schwierigkeitsgrades zum Nachdenken und zur Problemlösung, erhöhen die Studentenmotivation zum autonomen Lernen und stellen eine reale Sprachenform im konkreten Bereich dar. Das Ergebnis der Dissertation war auch, dass sich der Index der pädagogischen Polarität bei UE A auf einem höheren Wert beläuft als bei UE B. Ein höherer Wert des Indexes bei UE A drückt aus, dass sich die Lehrer-Schüler-Kontakte durch eine erhöhte Domination durch den Lehrer auszeichnen und dass der Lehrer eine höhere Anzahl strikter Anweisungen benötigt, um den Lernprozess zu leiten. UE B enthält hingegen mehr neutrale und weniger dominante Kontakte, die Arbeit verläuft kreativer, selbstständiger und handlungsorientierter. Die höhere Effizienz bei UE B wurde u. a. durch die Ergebnisse von didaktischen Tests bestätigt. Die Arbeit mit dem authentischen Material erfolgt selbstständiger und führt zu kreativeren Lösungen (Vávra 2015:56). Der Effekt der authentischen Materialien multipliziert sich durch erlebte Landeskunde und Einbeziehen möglichst vieler Sinne. Als Beispiel dafür kann das Mini-Projekt Kundenkarten dienen, dessen Ziel es war, auch persönliche Erfahrungen mit der Kundenkarte zu sammeln:

Das Decathlon-Logo befindet sich in der oberen linken Ecke, der Kundenname steht darunter. Der umseitige Barcode wird an der Kasse gescannt. Jetzt kommen wir zu den Vorteilen dieser Kundenkarte. Für jeden Einkauf erhält der Kunde Punkte, die später zu einem Rabatt werden. Der Kunde hat 90 Tage Zeit, um die Ware zurückzusenden. Auf der Karte werden alle Quittungen gespeichert, so dass der Kunde sie nicht aufbewahren muss. (Vostráková 2019:1)

Didaktische Ansätze dieser Art verfolgen projekt-, problem- und handlungsorientierte Ziele, die über die fachimmanenten Zielsetzungen der einzelnen Fächer hinausgehen. $\mathrm{Zu}$ ihren Prinzipien zählen Situationsbezug, Interdisziplinarität, Zusammenwirken von Kognitivismus und Interaktionismus sowie Individualisierung des Lerntempos. Von den vielen geeigneten Aktivitäten werden hier Stationenlernen, Learning-by-Doing und Mini-Projekte erwähnt. Produktive Fertigkeiten mit Ausrichtung auf die Wortfolge des deutschen Satzes können mit Hilfe von kreativem Schreiben entwickelt werden. Auch Exzerpieren, Beschreibung, Inhaltswiedergabe oder Erörterung mit der darauffolgenden Korrektur sind geeignete Formen des schriftlichen Ausdrucks. Im Zuge der Corona-Krise wird in der Fremdsprachendidaktik auch dem autonomen Lernen Aufmerksamkeit geschenkt, wobei zu selbstlernfördernden Faktoren u. a. expliziter Bezug auf Bekanntes, kleinschrittige Progression, Lehrprogression von allgemeineren zu detaillierteren Wissenselementen, hinreichende Explizitheit der Erklärungen und lernstrategische Vorschläge gehören.

\section{Abschluss}

Die Vorfeldbesetzung kann im DaF-Unterricht sowie beim autonomen Lernen anhand von authentischem Text- und Bildmaterial effizient geübt werden. Dies betrifft nicht nur das Wirtschaftsdeutsche, sondern auch das ganze Spektrum der Fachsprachen. Der Einsatz von Fachbüchern, Broschüren, Verträgen, Anträgen, Formularen, Handelsbriefen, Mails und Fotos entspricht der Realsituation, in der man im Ausland auch komplexe fremdsprachliche Texte verstehen muss, ohne ihren Schwierigkeitsgrad beeinflussen zu können. Dadurch wird die Aneignung einer wirklichkeitsgetreuen Vorfeldbesetzung während des Bachelorstudiums nicht zu einem langweiligen Syntax-Lernstoff.

Authentisches Material erfordert jedoch vom Lehrer eine gründliche Vorbereitung zusätzlicher Aufgaben zur Vorentlastung, um eine Überforderung der Studierenden zu vermeiden, sowie die Erstellung anschließender Übungsketten von kommunikativen Aufgaben aller Art: a. rezeptive (Richtig-Falsch-Aufgaben, Zuordnungsaufgaben u. a.); b. reproduktive (didaktische Spiele, Shadowing-Übungen im Dolmetschertraining oder auch ein kurzer „Redemitteldrill“ zum Einprägen der Schlüsselredemittel); c. produktive Aufgaben (Diskussionen, Rollen- und Planspiele, Erstellen von Werbetexten usw.). Der Lehrer sollte im Fremdsprachenunterricht statt langen eher kürzere, überschaubare Texte einsetzen, wie schon Blaasch in den 80er-Jahren (1985:122-123) betonte: 
„[...] dass die Schüler nicht von Anfang an mit einer ausführlichen Gesamtinformation konfrontiert werden - was ein mehr passives Schülerverhalten hervorrufen würde -, sondern sich selbst durch aktives Sprachhandeln aus vielen kleinen Texten mosaikartig ein Gesamtbild zusammensetzen.“

In der weiteren Forschung könnte die didaktische Grundhypothese auch in Bezug auf das Mittel- bzw. Nachfeld getestet werden: Je intensiver im DaF-Unterricht mit authentischen Materialien gearbeitet wird, desto wirklichkeitsgetreuer erscheint die Wortfolge des Schreibers/Sprechers am Ende seines Studiums. Die deutsche Wortfolge wird somit zu einem „Leuchtturm“, den man deutlich sehen kann, wenn es hell ist, doch dessen wahrer Zweck erst dann offenbar wird, wenn er den Studierenden im Labyrinth des Wirtschaftsdeutschen leuchtet.

\section{Literaturverzeichnis}

\section{Primärliteratur:}

BAUCHMÜLleR, Michael (2019): Prognose: Wirtschaft wächst nur noch um 0,8 Prozent. In: Süddeutsche Zeitung, Nr. 67, München, S. 1.

Čapková, Ladislava (2019): Geschäftskontakte und Teilnahme der westböhmischen Unternehmen an den bayerischen Messen. Bachelorarbeit ZČU, Plzeň. S. 45.

Čechová, Kateřina (2017): Krise mit der Nuss. Aufsatz zum Seminar KGS/NKP2. S. 1.

Hoyer, Niklas (2019): Auf einen Schlag mehr rausholen. In: Wirtschaftswoche, Nr. 3, Düsseldorf, S. 84.

N. N. (2019): DVB verkauft Luftfahrtgesellschaft. In: Frankfurter Allgemeine, Nr. 52, Frankfurt a. M., S. 20.

N. N. (2019): Regionalflughäfen zwischen Bangen und Bitten. In: Frankfurter Allgemeine, Nr. 52, Frankfurt am Main, S. 20.

N. N. (2019): Shell-Manager unter Verdacht. In: Frankfurter Allgemeine, Nr. 52, Frankfurt am Main, S. 22.

N. N. (2020): Wirtschaftsnachrichten. In: Plus, Nr. 1, Praha, S. 6.

Louven, Sandra (2019): Schattenseiten des Reisens. In: Handelsblatt, Nr. 75, Düsseldorf, S. 26.

VostŘÁKovÁ, Michaela (2019): Decathlon-Kundenkarte. Mini-Projekt. S. 1.

\section{Sekundärliteratur:}

Altmann, Hans / Hahnemann, Suzan (2010): Prüfungswissen Syntax. Göttingen.

Altmann, Hans / Hahnemann, Suzan (2007): Syntax fürs Examen. Studien- und Arbeitsbuch. Göttingen.

Beneš, Eduard (1977): Die funktionale Satzperspektive (Thema-Rhema-Gliederung) im Deutschen. In: HöFER, Karl-Heinz / PILling, Dieter/ SCHUBERT, Werner(Hrsg.): Germanistische Studientexte. Wort - Satz - Text. Leipzig, S. 122-127.

BlaAsch, Hans-Werner, 1985: „Wohnen in der Großstadt“. Überlegungen und Texte zu einem thematischen Baukasten für Fortgeschrittene. In: EDELHOFF, Christoph (Hrsg.) Authentische Texte im Deutschunterricht. Einführung und Unterrichtsmodelle. München.

DANEŠ, František (1974): Functional sentence perspective and the organization of the text. In: DANEš, František (Hrsg.): Papers on functional sentence perspective. Praha u. a., S. 106-128.

DürSCHEID, Christa (2007): Syntax. Grundlagen und Theorien. Göttingen.

EISENBERG, Peter (2001): Grundriss der deutschen Grammatik. Band 2: Der Satz. Stuttgart.

ErbEN, Johannes (1972): Deutsche Grammatik. Ein Abriß. München.

GuBER, Ines (2004): Die Thema-Rhema-Struktur informativer Texte. Baltmannsweiler.

Habermann, Mechthild / Diewald, Gabriele / Thurmair, Maria (2015): Duden. Grundwissen Grammatik. Fit für den Bachelor. Berlin.

HeLbig, Gerhard / BuschA, Joachim (2013): Deutsche Grammatik. Ein Handbuch für den Ausländerunterricht. München. 
Heringer, Hans Jürgen (1996): Deutsche Syntax. Dependentiell. Tübingen.

Jung, Walter (1968): Grammatik der deutschen Sprache. Leipzig.

Komenský, Jan Amos (1946). Didaktika analytická. Praha.

LUSCHER, Renate (2001): Übungsgrammatik für Anfänger. Ismaning.

Maroszová, Jana / VachKová, Marie (2009): Grundlagen der Syntax. Praha.

PeliKán, Jiř́i (2004): Základy empirického výzkumu pedagogických jevư. Praha.

PitTner, Karin / Berman, Judith (2015): Deutsche Syntax. Ein Arbeitsbuch. Tübingen.

RAMERS, Karl Heinz (2007): Einführung in die Syntax. Paderborn.

Schmid, Wilhelm (1977): Skizze der Kategorien und der Methode der funktionalen Grammatik. In: HöFER, Karl-Heinz / PILling, Dieter / SchuBerT, Werner (Hrsg.): Germanistische Studientexte. Wort - Satz - Text. Leipzig, S. 128-134.

SitTa, Horst (1998): Der Satz. In: DudenRedaKtion (Hrsg.): DUDEN, Grammatik der deutschen Gegenwartssprache. Mannheim u. a. S. 609-858.

VÁVRA, Zdeněk (2015): Suffixoide in umweltorientierten Texten - Implikationen für die Übersetzungsdidaktik. In: Lingua Viva, Nr. 20, České Budějovice, S. 56.

WeINRICH, Harald (1993): Textgrammatik der deutschen Sprache. Mannheim.

WöLLSTEIN, Angelika (2014): Topologisches Satzmodell. Heidelberg.

\section{Internetquellen:}

URL 1: BREINDL, Eva (2018): Grammis. Grammatisches Informationssystem. Fokuspartikel. https:// grammis.ids-mannheim.de/systematische-grammatik/408 [08.07.2020].

URL 2: Hennig, Mathilde (2018): Grammatikfragen.de. Vorfeldbesetzung - zwei Satzglieder im Vorfeld. https://grammatikfragen.de/showthread.php?1659-Ist-der-folgende-Satz-grammatikalischkorrekt-Einige-News-Artikel-schlie\%DFlich-haben-wir-gel\%F6scht\&p=3744 [09.07.2020].

URL 3: VÁVRA, Zdeněk (2020): Hospodářská němčina (nejen) pro bakaláře: KOncepty, KOmpetence, KOlokace. http://hdl.handle.net/11025/36870 [29.06.2020]. 